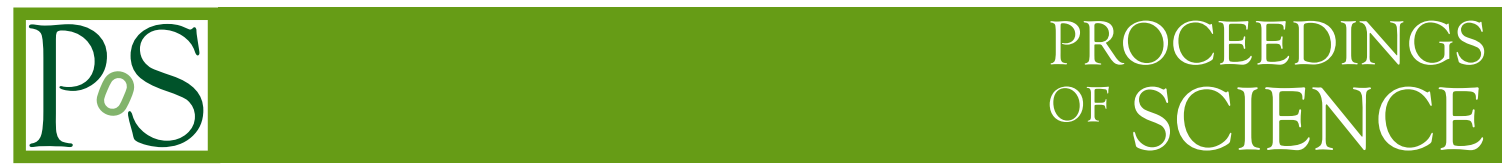

\title{
Charm production in fixed-target mode at $\mathrm{LHCb}$
}

\section{Frédéric Fleuret*}

Laboratoire Leprince-Ringuet (LLR), École polytechnique, CNRS/IN2P3, Palaiseau, France

E-mail: fleuretain2p3.fr

The first measurement of heavy-flavour production in a fixed-target mode at the LHC is presented. The production of $\mathrm{J} / \psi$ and $\mathrm{D}^{0}$ mesons is studied in collisions of protons with energies of $4 \mathrm{TeV}$ and 6.5 TeV incident on helium and argon nuclei at rest, corresponding to nucleon-nucleon centreof-mass energies of $\sqrt{s_{N N}}=86.6 \mathrm{GeV}$ and $\sqrt{s_{N N}}=110.4 \mathrm{GeV}$, respectively.

International Conference on Hard and Electromagnetic Probes of High-Energy Nuclear Collisions 30 September - 5 October 2018

Aix-Les-Bains, Savoie, France

${ }^{*}$ Speaker. 


\section{Introduction}

Heavy quarks and quarkonia, because of their mass - significantly larger than the $\Lambda_{Q C D}$ scale - are excellent tools to investigate the rich interface between the perturbative and non-perturbative domain of Quantum ChromoDynamics (QCD). Moreover, in the high temperature/density regime of QCD, they are decisive probes of the deconfined state of matter, the Quark Gluon Plasma (QGP) which can experimentally be studied in nucleus-nucleus collisions. Besides, the study of heavy flavour production in proton-nucleus collisions, where no QGP is formed, is needed for a correct interpretation of the results obtained in nucleus-nucleus collisions. This is crucial to establish a robust baseline in order to clearly identify phenomena specifically related to the QGP. In addition, several cold nuclear matter effects can be studied in proton-nucleus collisions, such as: Parton shadowing or anti-shadowing of the nuclear parton distribution functions (nPDF) in the target nucleus [1], saturation effects [2], interaction with comoving hadrons [3], and parton energy loss [4].

The LHCb detector [5] is a single-arm forward spectrometer covering the pseudorapidity range $2<\eta<5$, designed for the study of particles containing $b$ or $c$ quarks. The detector includes a high-precision tracking system consisting of a silicon-strip vertex detector (VELO) surrounding the interaction region, a large-area silicon-strip detector located upstream of a dipole magnet with a bending power of about $4 \mathrm{Tm}$, and three stations of silicon-strip detectors and straw drift tubes placed downstream of the magnet. Different types of charged hadrons are distinguished using information of two ring-imaging Cherenkov detectors and muons are identified by a system composed of alternating layers of iron and multiwire proportional chambers. A System for Measuring Overlap with Gas (SMOG) is associated to the silicon-strip vertex locator (VELO). The SMOG allows the injection of noble gas such as $\mathrm{He}, \mathrm{Ne}, \mathrm{Ar}, \mathrm{Kr}$ and $\mathrm{Xe}$.

In the following, the production of $\mathrm{J} / \psi$ and $\mathrm{D}^{0}$ mesons are studied in collisions of protons with energies of $4 \mathrm{TeV}$ and $6.5 \mathrm{TeV}$ incident on helium and argon nuclei at rest at centre-of-mass energies of $\sqrt{s_{N N}}=86.6 \mathrm{GeV}$ and $\sqrt{s_{N N}}=110.4 \mathrm{GeV}$, respectively.

\section{Data samples and selections}

The data samples have been collected under particular beam conditions where proton bunches moving towards the detector do not cross any bunch moving in the opposite direction at the nominal $p p$ interaction point. Events with $\mathrm{J} / \psi$ or $\mathrm{D}^{0}$ candidates must have a reconstructed primary vertex within the fiducial region $-200 \mathrm{~mm}<z_{P V}<200 \mathrm{~mm}$, where high reconstruction efficiencies are achieved and calibration samples available. In order to suppress residual $p p$ collisions, events with activity in the backward region are vetoed, based on the number of hits in VELO stations upstream of the interaction region.

Events with at least one primary vertex are selected where the primary vertex is reconstructed from at least four tracks in the VELO detector. The $\mathrm{J} / \psi$ candidates are obtained from two oppositely signed muons forming a good-quality vertex. The well-identified muons have a transverse momentum, $p_{\mathrm{T}}$, larger than $700 \mathrm{MeV} / c$ and are required to be consistent with originating from the primary interaction point. The kaon and pion from the $\mathrm{D}^{0}$ decay are required to come from a common displaced vertex. Tight requirements are set on the kaon and pion particle identification criteria. The $\mathrm{D}^{0}$ candidates are selected to have a decay time larger than $0.5 \mathrm{ps}$. The measure- 
ments are performed in the range of $\mathrm{J} / \psi$ and $\mathrm{D}^{0}$ transverse momentum $p_{\mathrm{T}}<8 \mathrm{GeV} / c$ and rapidity $2.0<y<4.6$. Acceptance and reconstruction efficiencies are determined using simulated $p \mathrm{He}$ and $p$ Ar events. In the simulation, $\mathrm{J} / \psi$ and $\mathrm{D}^{0}$ mesons are generated using PyTHIA 8 [6, 7]. The fourmomenta of the $\mathrm{J} / \psi$ and $\mathrm{D}^{0}$ daughters are then extracted and embedded into $p$ Ar or $p \mathrm{He}$ minimum bias events that are generated with the EPOS event generator [8] using beam parameters obtained from the data.

\section{3. $\mathrm{J} / \Psi$ and $\mathrm{D}^{0}$ production cross-sections}

The cross-section measurement is carried out for the $p$ He sample only, for which the luminosity determination is available. The luminosity is determined from the yield of electrons elastically scattering off the target $\mathrm{He}$ atoms [9] to be $\mathscr{L}_{p \mathrm{He}}=7.58 \pm 0.47 \mathrm{nb}^{-1}$. The measured $\mathrm{J} / \psi$ and $\mathrm{D}^{0}$ cross-sections per target nucleon within $y \in[2,4.6]$, after correction for the branching fractions $\mathrm{J} / \psi \rightarrow \mu^{+} \mu^{-}$and $\mathrm{D}^{0} \rightarrow K^{+} \pi^{-}$, are

$$
\sigma_{\mathrm{J} / \psi}=652 \pm 33 \pm 42 \mathrm{nb} / \text { nucleon }, \quad \sigma_{D^{0}}=80.8 \pm 2.4 \pm 6.3 \mu \mathrm{b} / \text { nucleon } .
$$

In order to compare to previous experimental results at different energies, both $\mathrm{J} / \psi$ and $\mathrm{D}^{0}$ crosssections are extrapolated to the full phase-space using PyтнiA 8. The full phase-space crosssections are

$$
\sigma_{\mathrm{J} / \psi}=1225.6 \pm 100.7 \mathrm{nb} / \text { nucleon }, \quad \sigma_{D^{0}}=156.0 \pm 13.1 \mu \mathrm{b} / \text { nucleon },
$$

where statistical and systematic uncertainties have been added quadratically and no systematic uncertainties due to the extrapolation are included. In addition, the $\mathrm{D}^{0}$ cross-section is scaled with the global fragmentation factor $f\left(c \rightarrow D^{0}\right)=0.542 \pm 0.024$ [10], in order to obtain the $c \bar{c}$ production cross-section $\sigma_{c \bar{c}}=288 \pm 24.2 \pm 6.9 \mu \mathrm{b} /$ nucleon. The last uncertainty reflects the limited knowledge of the fragmentation factor.

\section{Differential cross-sections and yields}

The $\mathrm{J} / \psi$ differential cross-sections per target nucleon obtained for the $p \mathrm{He}$ dataset, as functions of the rapidity in the centre-of-mass frame $y^{*}$ and transverse momentum $p_{\mathrm{T}}$, are shown in the two top plots of Fig. 1. These results are compared with HELAC-ONIA predictions [11], for $p p$ (blue dashed lines) and $p \mathrm{He}$ (green boxes) collisions. The $p p(p \mathrm{He})$ calculations use CT14NLO (nCTEQ15) parton densities. Since the predictions underestimate the measured total cross-section, they are rescaled by a factor 1.78 in Fig. 1 to compare the shape of the distributions. Data are also compared with phenomenological parametrisations, interpolated to the present data energies, based on Refs. [12, 13]. Solid and dashed red lines are obtained with linear and logarithmic interpolations, respectively, between experimental results obtained in $p \mathrm{Au}$ collisions at $\sqrt{s_{N N}}=38.7 \mathrm{GeV}, p \mathrm{C}$ collisions at $\sqrt{s_{N N}}=41.5 \mathrm{GeV}$ and $p p$ collisions at $\sqrt{s}=200 \mathrm{GeV}$. The differential yields of $\mathrm{J} / \psi$ as functions of $y^{*}$ and $p_{\mathrm{T}}$, obtained from $p$ Ar data, are also shown in Fig. 1. Since the luminosity measurement is not available, only differential distributions with arbitrary normalisation are shown.

The $\mathrm{D}^{0}$ differential cross-sections per target nucleon obtained for the $p \mathrm{He}$ dataset, as functions of $y^{*}$ and $p_{\mathrm{T}}$, are shown in Fig. 2. Since the HELAC-ONIA predictions underestimate the 

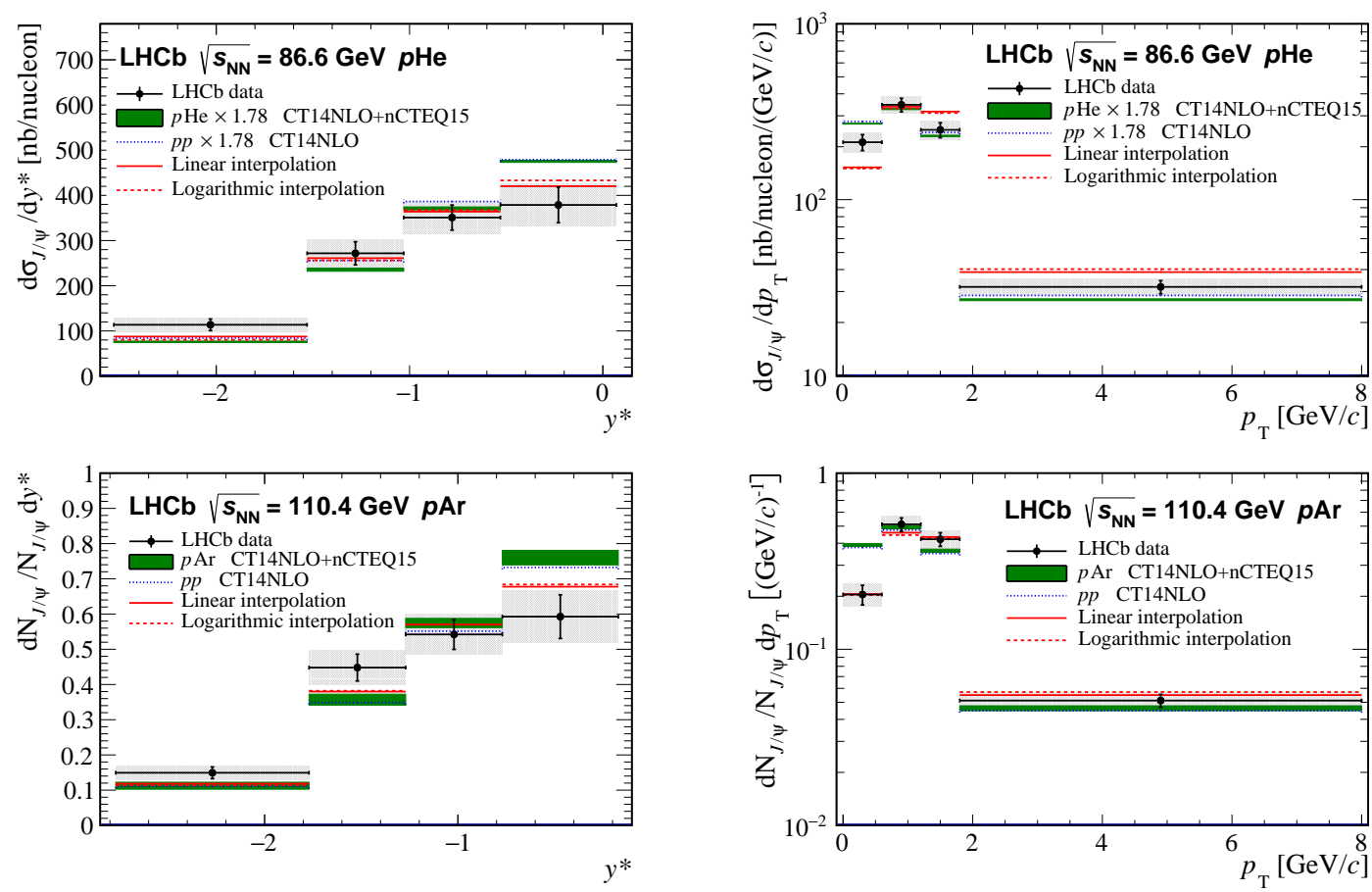

Figure 1: Differential $\mathrm{J} / \psi$ production cross-sections for (top) $p \mathrm{He}$ and differential $\mathrm{J} / \psi$ yields for (bottom) $p$ Ar collisions, as a function of (left) centre-of-mass rapidity $y^{*}$ and (right) transverse momentum $p_{\mathrm{T}}$. The data points mark the bin centres. The quadratic sum of statistical and uncorrelated systematic uncertainties are indicated by the vertical black lines. The correlated systematic uncertainties are indicated by the grey area. Theoretical predictions are described in the text.

measured total cross-section, they are rescaled by a factor 1.44 in Fig. 2 to compare the shape of the distributions. Differential yields, with arbitrary normalisation, of $\mathrm{D}^{0}$ as functions of $y^{*}$ and $p_{\mathrm{T}}$ obtained from $p$ Ar data are also shown.

In fixed-target configuration, the $\mathrm{LHCb}$ acceptance gives access to the large Bjorken- $x$ region of the target nucleon. In this region, because of the small number of nucleons in the helium nucleus, nuclear effects affecting $c \bar{c}$ pairs are expected to be small. On the other hand, as suggested in Refs. $[14,15]$, the intrinsic charm contribution, based on a valence-like parton distribution, can be substantial at large Bjorken- $x$ (typically $x \sim 0.3$ ). Using the approximation for $x$, the fraction of the nucleon momentum carried by the target parton,

$$
x \simeq \frac{2 m_{c}}{\sqrt{s_{N N}}} \exp \left(-y^{*}\right)
$$

where $m_{c}=1.28 \mathrm{GeV} / c^{2}$ is the mass of the $c$ quark, the Bjorken- $x$ range $x \in[0.17,0.37]$ is obtained for the most backward bin. In this range any substantial intrinsic charm contribution should be seen in the $p \mathrm{He}$ results. As shown in Fig. 1 and Fig. 2, no strong differences are observed between $p \mathrm{He}$ data and the theoretical predictions which do not include any intrinsic charm contribution. Therefore, within uncertainties, no evidence of substantial intrinsic charm content of the nucleon is observed in the data. 

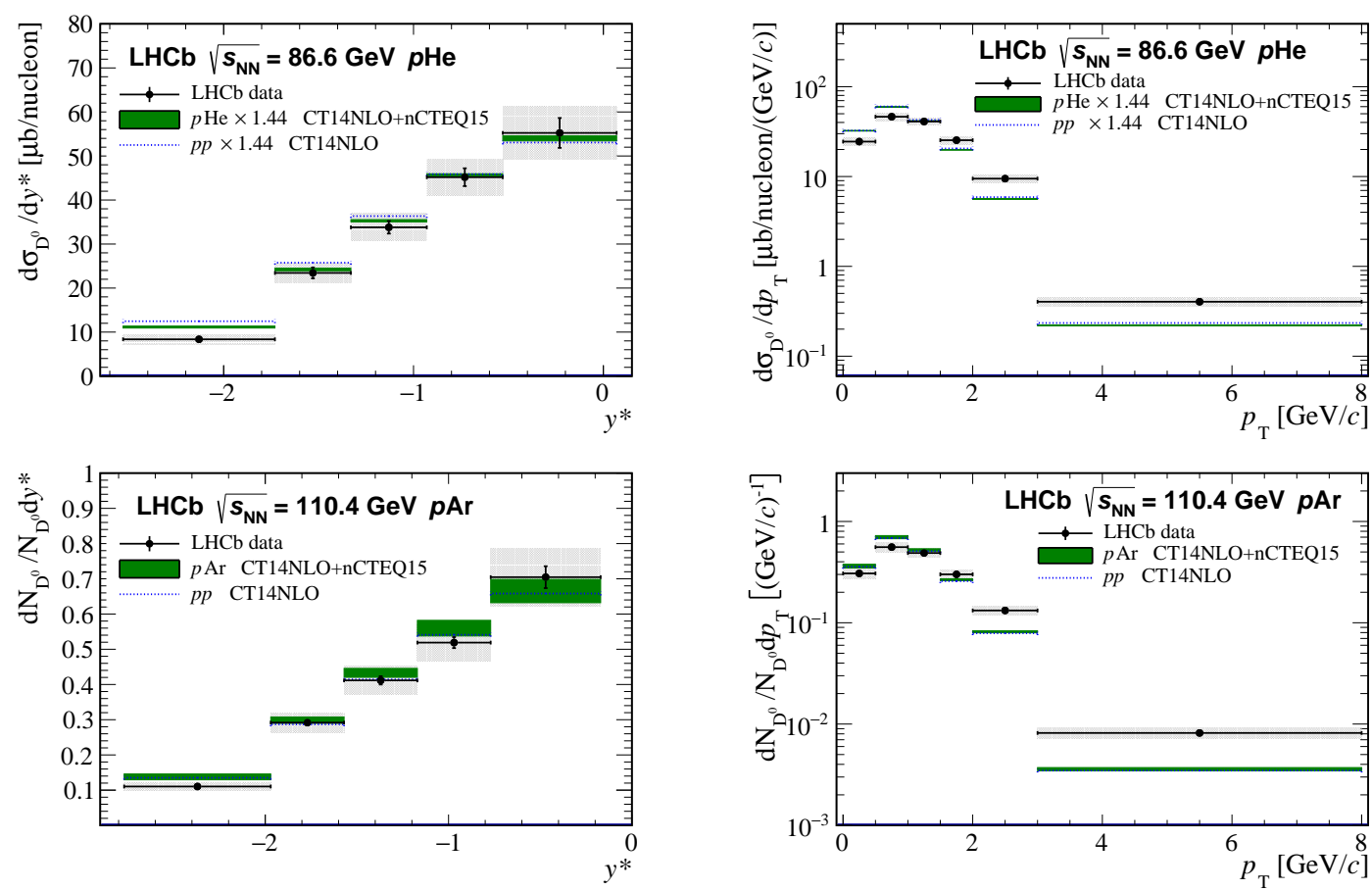

Figure 2: Differential $\mathrm{D}^{0}$ production cross-sections for (top) $p \mathrm{He}$ and differential $\mathrm{D}^{0}$ yields for (bottom) $p$ Ar collisions, as a function of (left) centre-of-mass rapidity $y^{*}$ and (right) transverse momentum $p_{\mathrm{T}}$. The data points mark the bin centres. The quadratic sum of statistical and uncorrelated systematic uncertainties are indicated by the vertical black lines. The correlated systematic uncertainties are indicated by the grey area. Theoretical predictions are described in the text.

\section{References}

[1] de Florian, D. and Sassot, R., Phys. Rev. D 69 (2004)

[2] J. L. Albacete and C. Marquet, Progress in Particle and Nuclear Physics 76 (2014) 1-42

[3] E. G. Ferreiro, Phys. Lett. B 749 (2015) 98

[4] Arleo, F. and Peigné, S., Phys. Rev. Lett. 109 (2012) 122301

[5] LHCb collaboration, A. A. Alves Jr. et al., JINST 3 (2008) S08005

[6] T. Sjöstrand, S. Mrenna, and P. Skands,Comput. Phys. Commun. 178 (2008) 852, arXiv:0710.3820

[7] T. Sjöstrand, S. Mrenna, and P. Skands, arXiv:hep-ph/0603175

[8] T. Pierog et al., Phys. Rev. C92 (2015) 034906, arXiv:1306.0121

[9] Phys. Rev. Lett. 121, 222001 (2018), arXiv:1808.06127

[10] L. Gladilin, Eur. Phys. J. C75 (2015) 19, arXiv:1404.3888

[11] J.-P. Lansberg and H.-S. Shao, Eur. Phys. J. C77 (2017) 1, arXiv:1610.05382

[12] F. Arleo and S. Peigné, JHEP 03 (2013) 122

[13] F. Arleo et al., JHEP 05 (2013) 155, arXiv:1304.0901

[14] J. Pumplin, H. L. Lai, and W. K. Tung, Phys. Rev. D75 (2007) 054029

[15] S. Dulat et al., Phys. Rev. D89 (2014) 073004 